\title{
Germanica
}

GERMANICA

$41 \mid 2007$

Le livret d'opéra en langue allemande au $\mathrm{XX} \mathrm{X}^{\mathrm{e}}$ siècle : ruptures et reprises

\section{À propos de Turandot de Ferruccio Busoni}

Une poétique de l'opéra classique et romantique?

Gozzis fiabe und Busonis Turandot und Arlecchino (1917)

Jean-François Candoni

\section{(c) OpenEdition}

Journals

Édition électronique

URL : http://journals.openedition.org/germanica/477

DOI : 10.4000/germanica.477

ISSN : 2107-0784

Éditeur

Université de Lille

Édition imprimée

Date de publication : 1 décembre 2007

Pagination : 19-34

ISBN : 978-2-913857-20-9

ISSN : 0984-2632

Référence électronique

Jean-François Candoni, «À propos de Turandot de Ferruccio Busoni », Germanica [En ligne], 41 | 2007 mis en ligne le 01 décembre 2009, consulté le 06 octobre 2020. URL : http://journals.openedition.org/ germanica/477 ; DOI : https://doi.org/10.4000/germanica.477

Ce document a été généré automatiquement le 6 octobre 2020.

(c) Tous droits réservés 


\title{
À propos de Turandot de Ferruccio Busoni
}

\author{
Une poétique de l'opéra classique et romantique? \\ Gozzis fiabe und Busonis Turandot und Arlecchino (1917)
}

Jean-François Candoni

1 En choisissant de composer son troisième opéra sur une adaptation de Turandot, une fiaba teatrale du Vénitien Carlo Gozzi, Ferruccio Busoni se place résolument dans une démarche de réappropriation de l'esthétique romantique, inaugurée par son premier opéra, Die Brautwahl (Le Choix d'une fiancée), inspiré quant à lui du récit homonyme d'E.T.A. Hoffmann. Aussi paradoxal que cela puisse sembler au premier abord, l'utilisation par le compositeur et librettiste d'une fiaba teatrale vénitienne témoigne en effet d'une volonté de renouer avec le romantisme littéraire à la Hoffmann plus que d'un désir de s'inscrire dans une tradition opératique italienne: Busoni, qui avait brièvement pensé faire de Turandot un opéra italien ${ }^{1}$, se décide finalement pour la langue allemande alors que, comme le remarque Antony Beaumont ${ }^{2}$, le théâtre de Zurich, où devait être créée l'œuvre le 11 mai 1917, ne mettait pas d'obstacle à la représentation d'une œuvre en italien. Le choix de la langue allemande est parfaitement délibéré et n'est pas commandé par les circonstances: le compositeur germano-italien se situe ainsi dans la lignée de la réception de l'œuvre de Gozzi en Allemagne, qui a joué un rôle déterminant dans la constitution de l'esthétique romantique.

2 Si Busoni a jeté son dévolu sur l'auteur vénitien, sur lequel il avait déjà eu l'occasion de travailler en composant une musique de scène pour des représentations de la version allemande de Turandot réalisée par Karl Vollmoeller et mise en scène par Max Reinhardt en 1911, c'est sans doute parce qu'il y voyait un moyen de renouer avec l'esprit hoffmannien de son tout premier opéra : le fameux écrivain et musicien, dont plusieurs récits sont imprégnés de l'univers drolatique et de l'imaginaire merveilleux de Gozzi, écrit dans Le Poète et le Compositeur (1819-1821): "dans ses contes dramatiques, [Gozzi] a entièrement réalisé ce que j'attends d'un librettiste d'opéra, et il est incompréhensible que cette riche mine d'excellents sujets d'opéra n'ait pas été plus 
exploitée jusqu'à présent ${ }^{3}$ ». Non seulement Busoni était un admirateur inconditionnel d'Hoffmann, écrivain pourtant peu prisé en ce début de $\mathrm{xx}^{\mathrm{e}}$ siècle, mais en outre, sa propre conception de l'opéra entrait parfaitement dans le cadre d'une lecture «romantique » et hoffmannienne de l'œuvre de Gozzi mettant l'accent sur l'aspect irréel des fiabe, sur le rôle qu'y joue le surnaturel, mais aussi sur la mise à distance du tragique et du pathos imposée par l'omniprésence d'interventions ironiques et grotesques, essentiellement confiées aux masques de la commedia dell'arte.

Parce qu'il considère, à l'instar d'E.T.A. Hoffmann, que la musique est le langage de l'absolu, qu'elle « ouvre à l'homme un royaume inconnu, totalement étranger au monde sensible qui l'entoure ${ }^{4}$ », Busoni met l'accent sur la dimension spirituelle de la musique d'opéra, excluant ainsi qu'elle puisse être trop étroitement associée aux mots et que la mélodie puisse se dissoudre dans une sorte de prose musicale réaliste: " la parole chantée sur scène restera toujours une convention et un obstacle à tout effet authentique ${ }^{5}$ ». En reprenant le topos romantique d'une musique qui serait le langage de l'indicible et de l'absolu, Busoni entend faire un sort à la fois au vérisme et à Wagner, dont il fait une lecture non pas romantique, mais résolument réaliste. Par une ironie de l'histoire, c'est en instrumentalisant le théâtre de Gozzi qu'il cherche à se démarquer de Wagner, alors que son illustre aîné avait lui-même fait ses débuts à l'opéra (Les Fées / Die Feen) avec une très hoffmannienne adaptation de La Femme serpent (La donna serpente) de Gozzi. Mais il y a une différence fondamentale entre l'adaptation de Gozzi par Wagner et celle de Busoni : la première gomme en effet tous les éléments comiques empruntés à la commedia dell'arte pour tenter de retrouver l'authenticité d'un conte perçu comme une sorte de mythe en miniature ${ }^{6}$, la seconde cherche à chaque instant à mettre en avant l'ironie et la distanciation.

Busoni, qui rédigeait lui-même ses livrets, prêtait une attention toute particulière au choix de ses sources: pour lui, seuls entraient en ligne de compte les sujets qui appellent obligatoirement la musique pour trouver leur plein accomplissement. Or, les sujets utilisés dans les opéras considérés comme véristes au sens large du terme (il cite comme exemple La Tosca de Victorien Sardou et La Dame aux camélias d'Alexandre Dumas) fonctionnent selon lui parfaitement dans le cadre du théatre parlé et peuvent très bien se passer d'un habillage musical qui ne leur apporte au fond rien de déterminant. Pour le compositeur, il s'agit donc de trouver des sujets littéraires qui non seulement ne se rebellent pas contre l'intervention du compositeur, mais qui soient en outre réellement en attente de musique pour trouver leur véritable sens :

Tandis qu'il existe pour le théâtre parlé un choix de sujets pratiquement illimité, il apparaît que, pour l'opéra, les seuls sujets appropriés sont ceux qui ne peuvent exister ni trouver leur plus parfaite expression sans musique, les sujets qui exigent la musique et ne trouvent leur achèvement que par elle. ${ }^{7}$

Busoni était convaincu que les fiabe de Gozzi appelaient naturellement la musique, qui seule devait leur permettre de révéler leurs qualités profondes. Sur ce point, le compositeur germano-italien rejoint de façon inattendue les positions de Friedrich Schlegel. On ne sait pas si Busoni avait eu connaissance des passages consacrés aux fiabe gozziennes par l'éditeur de l'Athenaeum, mais celui-ci affirmait que leur dimension poétique latente ne pouvait se révéler totalement à cause de l'absence de musique, ce qui était déjà une invitation implicite à composer, sinon un opéra, du moins une musique de scène :

Dans les contes populaires fantastiques de Gozzi, dans ses pièces pleines de merveilleux et de spectaculaire, nous pouvons voir une force d'invention 
véritablement poétique; mais sans les qualités musicales, sans la parure de la fantaisie par lesquelles la poésie qu'elles recèlent pourrait se manifester complètement et produire tout son effet. ${ }^{8}$

6 Busoni a particulièrement été attiré, en lisant les fiabe de Gozzi, par leur absence de pathos et leur goût pour le jeu et la mise à distance, voire l'ironie, résultant de la confrontation entre une intrigue sérieuse, empruntée au conte, et les interventions comiques, voire grotesques, des masques de la commedia dell'arte. Une fois de plus, sa lecture de Gozzi se révèle étonnamment proche de celle des romantiques allemands - et elle se situe à l'opposé du projet de Schiller de mettre l'accent sur la caractère tragique de Turandot. Tandis que Friedrich Schlegel avait été séduit par l'aspect « décoratif » des fiabe (il parle à leur sujet de "Decorationspoesie »"), et qu'August Wilhem Schlegel avait mis l'accent sur leur refus de toute espèce de réalisme ou de vraisemblance ${ }^{10}$, Busoni souligne leur dimension irréelle :

Le sentiment que, même dans les scènes qui confinent au tragique, il s'agit toujours d'un jeu, disparaît complètement chez Schiller. Ce sont avant tout les masques, que les Italiens connaissent bien, qui jettent un pont entre le public vénitien et l'Orient de fiction montré sur la scène, anéantissant ainsi l'illusion qu'il s'agit d'événements réels. ${ }^{11}$

7 À l'inverse de Schiller, Busoni cherche donc, dans son adaptation de Turandot, à gommer tout ce qui pourrait la rapprocher d'une tragédie et insiste sur l'humour, le grotesque et l'ironie, comme s'il ne fallait jamais prendre au sérieux ce qui se déroule sur scène, même (et surtout) dans les passages où la cruauté de la princesse chinoise produit ses effets les plus dévastateurs. Il ne fait d'ailleurs au fond que prendre au pied de la lettre les allégations de Gozzi lui-même, lequel parlait à propos des contes mis en scène dans les fiabe $d^{\prime}$ ' une ineptie puérile ${ }^{12}$." Les personnages de la fiaba eux-mêmes considèrent les épreuves imposées par la princesse chinoise à ses prétendants comme une « fable », comme un « jeu ${ }^{13} »$.

8 Le compositeur voit dans ce principe du jeu, de la distance ironique par rapport aux événements sanglants liés au comportement de Turandot, un principe esthétique qui lui permet de se démarquer des grands modèles dominants de l'opéra du début du $\mathrm{xx}^{\mathrm{e}}$ siècle, le vérisme italien et le wagnérisme allemand, un moyen d'inventer (ou de réinventer) une forme d'opéra qui ne repose ni sur le principe d'identification (le vérisme), ni sur la fascination hypnotique (Wagner), mais plutôt sur une forme d'intérêt qui soit suffisamment distancié pour préserver la liberté esthétique du spectateur. Paradoxalement, la conception de l'opéra qui sous-tend Turandot nous ramène à Schiller, non à pas l'adaptateur de Gozzi, mais cette fois-ci à l'essayiste, qui affirmait dans ses Lettres sur l'éducation esthétique de l'homme (1795) que «l'homme doit seulement jouer avec la beauté » et qu'il «doit jouer seulement avec la beautée ${ }^{14}$ ». En quête de ce qu'il appelait le " jeune classicisme » («Junge Klassizität»), Busoni entendait que la musique soit l'expression d'une liberté totale, qu'elle échappe au formalisme étroit aussi bien qu'à l'esprit de système wagnérien. C'est pourquoi, lorsqu'il essaie de définir dans quelles situations le recours à la musique est indispensable au théâtre, il évoque d'une part l'irruption du surnaturel (curieusement, il a choisi avec Turandot la seule fiaba dont le surnaturel soit totalement absent - sans doute pensait-il qu'il revenait à la musique d'introduire cette présence du supranaturel que le texte n'évoquait pas, mais qu'il laissait malgré tout vaguement pressentir), et d'autre part l'idée $d u$ «jeu absolu, du travestissement divertissant, de la scène en tant que 
simulation ostensible et revendiquée, dans l'idée de la bouffonnerie et de l'irréalité comprises comme antipodes du sérieux et de la vérité de la vie. ${ }^{15}$ »

Cette vision de l'art conçu comme jeu est thématisée dans le livret lui-même, qui met en scène la volonté de dépasser, par le truchement du jeu, le pathos et le tragique d'un sentiment amoureux qui conduit à la mort afin d'aboutir à une relation de couple équilibrée, reposant sur l'estime et le respect mutuel des deux partenaires. Si Kalaf parvient si aisément à résoudre les énigmes posées par Turandot, c'est parce qu'il comprend qu'il est question d'art, et donc de jeu («je joue ma vie contre un poème ${ }^{16}$ » s'exclame-t-il). Comprendre quels sont les enjeux réels de l'art, c'est apprendre à distinguer l'image sensible du réel, c'est prendre conscience que l'art n'est qu'un jeu, un jeu sérieux, mais tout de même un jeu. Kalaf devra donc apprendre à dépasser la fascination irrationnelle exercée sur les prétendants par le portrait de la princesse. C'est en voyant son portrait (comparé au visage de la Méduse) au début du premier acte qu'il tombe amoureux, mais d'un amour qui n'est qu'oubli de soi et perte d'identité : il oublie la noble mission dont il s'est chargé lui-même (restaurer la gloire et la dignité de la famille royale dont il est issu), puis il se présente aux épreuves comme un candidat anonyme et refuse de révéler son nom à l'empereur Altoum, signe étrange d'une aliénation librement consentie. Cet amour qui s'empare soudainement de lui est une pulsion d'autodestruction. Il relève pour Busoni, d'un état pathologique qui rappelle, transposé dans la sphère musicale, l'effet recherché par l'esthétique vériste ou l'art wagnérien. On reconnaît d'ailleurs ici les grandes lignes de l'argumentation développée par Nietzsche contre « le cas Wagner ${ }^{17}$ ». Si l'on peut voir dans ce portrait qui hypnotise littéralement Kalaf un hommage en forme de clin d'œil au début de La Flûte enchantée de Mozart (et donc un hommage discret au classicisme viennois), sa signification est cependant différente: Tamino tombe amoureux de Pamina en raison des vertus universelles qu'elle incarne ${ }^{18}$, et pas parce qu'il succombe, comme les prétendants de Turandot, à l'emprise d'une sorte de fascination morbide qui est au final plus proche de celle qu'exerce sur Senta le portrait du Hollandais dans Le Vaisseau fantôme de Wagner. Il faut plutôt voir dans cette inexplicable fascination émanant du portrait l'allégorie d'une forme de musique pathologique, d'un art qui agit sur les nerfs de l'auditeur, si bien que l'on pourrait, en paraphrasant Kalaf lorsqu'il se demande, avant de l'avoir vu, «comment un portrait pourrait être mortel ${ }^{19}$ ", poser la question de savoir « comment une musique pourrait être une névrose ? " Busoni, qui estimait (sans doute pensait-il à Tristan et Isolde) qu'un duo d'amour était obscène sur une scène d'opéra, exprime très clairement, dans sa préface à la partition du Doktor Faust, sa méfiance envers le pouvoir hypnotique et érotique de la musique :

La musique sensuelle ou "sexuelle» (qui, consiste en une sorte d'obstination, d'ivresse sonore et joue ainsi sur la gamme des nerfs) n'a pas sa place ici, cela vient de façon naturelle de l'essence même de cet art, qui est purement abstrait ${ }^{20}$.

Une musique sensuelle qui ignore l'essence abstraite et spirituelle de l'art des sons est pour Busoni le produit d'une conception hétéronome de la musique qui tente de faire dépendre le discours musical de réalités non musicales: dès lors que la musique cherche à devenir langage ou à désigner des éléments du réel, elle s'approche de cette musique à programme qui cherche indûment à s'emparer de la réalité sensible. Il voit dans ce principe esthétique de l'hétéronomie musicale une dénaturation de l'essence même de l'art des sons et il préfère accorder la prééminence à la musique sur le texte, au compositeur sur le librettiste. 
11 À cet égard, Busoni nous paraît bien éloigné du Literaturoper tel que le conçoivent des personnalités aussi différentes que Richard Strauss ou Alban Berg. La pièce de Gozzi subit une série de remaniements drastiques qui ne sont pas sans rappeler la façon dont travaillaient les librettistes de la première moitié $d u$ XIXe siècle : les cinq actes sont ramenés à deux, l'intrigue que la pièce vénitienne développe autour du personnage d'Adelma, la servante de Turandot secrètement amoureuse de Kalaf, est tellement simplifiée que, comme c'est souvent le cas dans l'opéra traditionnel, ses motivations en deviennent incompréhensibles. Mais l'exemple le plus parlant de ce refus du Literaturoper est sans doute la façon dont Busoni transforme les tirades du Calaf de la fiaba justifiant, devant l'empereur, les raisons pour lesquelles il veut se soumettre au jeu des épreuves, en un simple «slogan», prononcé à quatre reprises : «La mort ou bien Turandot ${ }^{21} »-$ ce sont, à peu de choses près, ses seules paroles prononcées au cours de cette scène, conçue comme un quatuor. Le compositeur s'en explique, quelques années plus tard, dans son Ébauche de préface à la partition du Doktor Faust, lorsqu'il affirme que "le slogan remplace à l'opéra la tirade du drame parlé22 " (le terme de slogan est pris dans un sens large puisqu'il inclut également le langage scénique), renouant ainsi avec une pratique du livret d'opéra qui, de Fidelio de Beethoven à Attila de Verdi en passant par La Muette de Portici d'Auber, est une constante de la production européenne de la première moitié $\mathrm{du} \mathrm{xIX}^{\mathrm{e}}$ siècle. On notera toutefois chez Busoni cette différence majeure avec les habitudes du siècle précédent : ses livrets ne misent pas uniquement sur l'enchaînement des situations dramaticomusicales, mais apportent un soin tout particulier à la qualité littéraire du texte et au sens symbolique des paroles prononcées. Il est indiscutable que l'œuvre de Wagner a posé dans ce domaine des exigences sur lesquelles il paraît difficile de revenir.

12 La méfiance de Busoni envers la littérarisation de l'opéra s'explique par les mêmes raisons que sa détestation du pathétisme exacerbé du vérisme et de l'ivresse sonore wagnérienne : il voit dans ces phénomènes différents aspects d'une même réalité (on pourrait dire: d'un même réalisme), d'une volonté de soumettre la musique à des éléments hétéronomes qui nient sa nature purement spirituelle. Tous ces phénomènes sont condamnés au nom du "jeune classicisme » ("Junge Klassizität»), concept sous lequel il réunit quelques-uns de ses grands principes esthétiques, en particulier le "renoncement à la sensualité", l'abandon du subjectivisme et l'« effacement de l'auteur devant l'œuvre ${ }^{23} »$. Cela ne signifie pas pour autant qu'il se rallie au formalisme musical défendu par Eduard Hanslick dans son fameux essai Du beau dans la musique (1854). Pour Busoni, la musique, en particulier à l'opéra, doit être la manifestation sensible d'une réalité spirituelle supérieure (« une partie des vibrations de l'univers ${ }^{24}$ »), et non un simple jeu « de formes sonores en mouvement ${ }^{25}$ » n'ayant d'autre contenu ou objet que lui-même, et dont les sentiments du sujet se seraient absentés :

«La musique absolue » [il est fait allusion ici au formalisme musical de Hanslick] est un jeu de formes sans programme poétique dans lequel la forme joue le rôle essentiel. Mais la forme est justement à l'opposé de la musique absolue [i.e. la musique où s'exprime l'absolu], qui a reçu le privilège divin de planer librement, indépendamment de toute détermination matérielle ${ }^{26}$.

13 Cette voie médiane entre le formalisme radical de Hanslick et l'esthétique réaliste que Busoni prête à Wagner (sa vision du maître de Bayreuth n'est pas fausse, mais elle tend à réduire son esthétique à une seule de ses composantes) est une façon pour le compositeur de renouer avec le romantisme musical d'E.T.A. Hoffmann et celui de Tieck et Wackenroder ${ }^{27}$. Si Busoni a pu être accusé de futurisme par Hans Pfitzner (sans 
doute à cause de son projet, jamais mis en œuvre, de composer une musique utilisant les tiers de ton), il faut bien reconnaître que ses positions esthétiques ne sont pas complètement nouvelles. Theodor W. Adorno remarquait déjà en 1930, dans un développement consacré à Arlecchino et à Turandot, que « toute sa production se situe à la lisière du romantisme. ${ }^{28}$ »

Mais si on les observe attentivement, on découvre que les positions théoriques de Busoni sont très proches de celles que défendait, au milieu du XIX ${ }^{\mathrm{e}}$, le musicologue et historien de la musique August Wilhelm Ambros. Certes, le compositeur ne se réfère pas explicitement, dans ses différents essais, aux théories esthétiques du célèbre musicologue, mais il est évident qu'il en avait pris connaissance puisque l'essentiel des motifs musicaux « orientaux » présents dans la partition de Turandot (qu'il s'agisse de la musique de scène ou bien de l'opéra, qui réutilise le matériau musical de la première) sont empruntés à l'Histoire de la musique (Geschichte der Musik, 1862-1878) d'Ambros ${ }^{29}$. Comme Busoni, Ambros se méfiait d'une musique trop ancrée dans la sensualité et l'excitation nerveuse ${ }^{30}$, il réfutait l'idée que sa fonction soit de désigner le réel («la musique n'a pas de mots pour dire les faits $\left.{ }^{31} »\right)$, mais il n'en restait pas moins extrêmement sceptique envers les positions formalistes, qui cherchent à réduire l'art des sons à une série d'«arabesques sonores" et donc à sa composante purement formelle ${ }^{32}$, estimant lui aussi que l'essence de la musique réside dans des idées poétiques que seul l'art des sons est capable d'exprimer : «La composante spirituelle de l'œuvre d'art, sa composante idéale est l'étincelle prométhéenne prise au ciel, qui donne vie à la belle forme sonore. ${ }^{33}$ " Ou bien on pourrait parler, en reprenant la définition de l'art donnée par Kalaf dans la troisième énigme, de «baiser donné à la terre par la grâce divine. ${ }^{34}$ » Cette conception de la musique qui met en avant sa dimension spirituelle, sa capacité à dire l'indicible, son irréductibilité au langage des mots et donc son rejet de toute espèce de réalisme musical, place Busoni non pas du côté de la modernité, mais plutôt dans la continuité d'une tradition ancienne qui associe la musique, et en particulier l'opéra, au merveilleux. Comme le note Carl Dahlhaus,

Il est pour ainsi dire dans la nature même du drame musical, dans la mesure où il s'efforce d'être un drame conçu à partir de la musique, de traiter de sujets merveilleux. La théorie de l'opéra a toujours été, que ce soit au XVII ${ }^{e}$ ou au XVIII siècle, mais également dans la période qui va du romantisme d'E.T.A. Hoffmann jusqu'à Busoni en passant par Wagner, une esthétique du "merveilleux", du "meraviglioso" - à l'opposé du "vraisemblable" qui, depuis Aristote, est exigé par la poétique du théâtre parlé. ${ }^{35}$

Dans le sillage de l'esthétique romantique, Busoni a d'ailleurs intégré des éléments de sa réflexion sur la poétique de l'opéra, centrée sur la problématique des rapports entre le spirituel et le monde sensible, au cœur même de son œuvre artistique. Les trois énigmes de Turandot, qui portent respectivement sur l'entendement, sur la coutume et sur l'art, font de cet opéra une sorte d'allégorie de la conception de l'art selon Busoni (dans la fiaba italienne, les trois énigmes portent sur le soleil, l'amour et le lion de Venise). Mais, curieusement, on a l'impression que le librettiste s'est amusé à inverser les données, en ce sens où les réponses sont les véritables questions (qu'est-ce que l'entendement? qu'est-ce que la coutume? qu'est-ce que l'art?), tandis que les questions posées par la princesse font figure d'explication visant à définir ces trois notions. L'entendement est présenté comme une force qui pousse l'homme à échapper à sa condition matérielle et à tendre vers la lumière et la spiritualité («Qu'est-ce qui 
rampe sur le sol, s'envole vers le ciel, / Qu'est-ce qui tâtonne dans l'obscurité et allume des lumières [...] ?) $»^{36}$, ce qui renvoie d'une part à une problématique centrale du livret, celle des exigences intellectuelles que la princesse se fixe à elle-même et à ses prétendants, mais correspond d'autre part à l'une des fonctions essentielles assignées à l'art par Busoni. La présence d'esprit de Kalaf, son acuité intellectuelle sont ce qui lui permet d'échapper à l'envoûtement irrationnel qu'exerce la princesse sur les jeunes hommes, même s'il manque lui aussi d'y succomber, totalement ébloui par la beauté qui s'offre à lui, et de ne pas pouvoir résoudre la troisième énigme («j'étais pris d'étourdissement $\left.{ }^{37} »\right)$, celle justement où il est question de l'art.

La deuxième énigme, qui présente la coutume comme une loi tyrannique, mais soumise à la péremption temporelle, peut être mise en parallèle avec l'aspect purement technique et formel de l'œuvre d'art, dont le compositeur nous dit dans son essai Pour une nouvelle esthétique musicale qu'il s'agit là de la composante éphémère de l'œuvre (celle qui fait sa modernité), tandis que l'esprit et le sentiment en constitueraient l'élément de permanence :

L'esprit d'une œuvre d'art, la densité de sentiments, l'élément humain qui est en elle - toutes ces choses restent sans que leur valeur change à travers les époques; la forme qu'ont prise ces trois éléments, les moyens qui les exprimaient et le goût dans lequel l'époque de leur genèse les a enveloppés sont éphémères et vieillissent vite. $^{38}$

17 La troisième énigme enfin, celle qui donne rétrospectivement leur sens aux deux autres, propose une définition de l'art qui confirme que l'on peut aborder le livret dans une perspective romantique d'autoréflexion de l'œuvre d'art. Le commentaire de la princesse met l'accent sur la dimension temporelle de l'art (son ancrage dans le passé lointain de la communauté humaine), ce qui renvoie à la réflexion développée par Busoni sur son rapport complexe à la tradition, dans laquelle il s'inscrit résolument (le «jeune classicisme », qui veut que le compositeur renonce au travail thématique, cher à Wagner, au profit de la mélodie) tout en cherchant à ouvrir sur des perspectives d'avenir. Mais surtout, on relève une fois de plus la référence explicite au romantisme de Tieck, Wackenroder et Hoffmann dans la conception d'un art «sur lequel règnent des élus solitaires ${ }^{39}$.»

La troisième énigme est à peine résolue qu'Altoum ordonne que l'on joue de la musique : pour le pianiste et compositeur, la musique constitue l'art par excellence, ce qui justifie sa théorie d'un opéra qui serait une « œuvre d'art totale musicale ${ }^{40}$ », non pas au sens de l'œuvre d'art totale wagnérienne, mais au sens d'une œuvre musicale qui réunirait toutes les formes de musique indépendamment des conventions relatives à leur destination (opéra, église ou concert) et aux moyens sonores requis (voix, instruments...).

19 Aux trois questions de la princesse fait ensuite écho l'énigme posée à Turandot par le prince inconnu à la toute fin du premier acte. Cette dernière repose quant à elle sur un mouvement qui va dans le sens inverse des énigmes posées par la princesse, destinées à éprouver les qualités intellectuelles des prétendants, puisque Kalaf attend de celle qu'il aime qu'elle aille de l'abstraction intellectuelle vers l'individualité réelle: mettre un nom sur le prince inconnu, qui n'est au fond que le dernier d'une longue série de prétendants anonymes, c'est lui donner une épaisseur temporelle (en même temps que son nom se révèle une histoire, celle d'un fils de roi victime des vicissitudes de la guerre) et spatiale (la recherche de ses origines géographiques), c'est éprouver de la 
sympathie pour un destin personnel, c'est au fond voir une individualité derrière ce qui n'était au premier abord qu'une abstraction. C'est pourquoi résoudre l'énigme du nom est nécessaire pour que la jeune vierge froide et apparemment inhumaine puisse sortir de son enfermement dans la stérilité de l'abstraction intellectuelle: à l'instar de Cherestani, l'héroïne de La donna serpente transformée en statue de pierre, puis délivrée et rendue à l'état de femme par l'amour de Farruscad, Turandot accède pleinement, à la fin de l'opéra, à cette harmonie que Kalaf avait cru entrevoir dans son portrait («Barak, tout en elle est harmonie $\left.{ }^{41} »\right)$.

Le danger d'une attitude purement intellectuelle qui ignorerait le sensible est suggéré sur un mode à la fois ironique et grotesque par le personnage de Truffaldino, le chef des eunuques chargés de la protection de Turandot. Sûr de sa supériorité intellectuelle présumée, il méprise les hommes qui sont à la recherche d'une compagne (il ironise sur ceux qui, selon lui, ne sont pas capables de marcher sur une seule jambe), ce qui fait ainsi écho, sur le mode de la caricature, au refus de la princesse d'épouser un homme. Si la supériorité intellectuelle de Turandot est validée par l'incapacité des prétendants à résoudre ses énigmes, celle de Truffaldino, qui prétend que «la chance du castrat, c'est la supériorité de son esprit ${ }^{42} »$, est constamment prise en défaut. C'est le cas notamment au second acte lorsque, chargé par sa maîtresse de découvrir le nom du prince inconnu, il revient à elle en prétendant s'être brillamment acquitté de sa mission avant de reconnaître (sans vraiment avoir conscience d'avoir échoué) qu'il n'a su tirer du prince endormi d'autres paroles que cette phrase, déjà prononcée à plusieurs reprises à la fin du premier acte : «La mort, la mort ou bien Turandot ${ }^{43}$ » La cécité intellectuelle de Truffaldino, esprit mutilé et impuissant à agir, n'est qu'un reflet grotesque et disgracieux de l'attitude de la princesse, ou du moins de ce qui la guette si elle persiste dans son aveuglement: murée dans l'isolement stérile de son arrogance intellectuelle, elle refuse de se réconcilier avec la vie réelle, avec le monde sensible et avec le cours inéluctable du temps qu'elle parait vouloir immobiliser en refusant de devenir femme et mère - le vieil empereur Altoum attend désespérément que sa fille accepte un époux pour pouvoir se retirer et céder sa place, qui revient naturellement aux générations à venir. Transposée au niveau de la poétique de l'opéra qui se constitue progressivement dans Turandot, cette mise en garde nous rappelle que si l'art est certes un jeu, il ne saurait se réduire à un jeu purement intellectuel, abstrait, gratuit et stérile. Il y a au contraire, dans l'idée de l'art défendue par Busoni, un lien avec le sacré. Le sérieux du jeu à l'opéra, qui se révèle dans sa dimension quasi religieuse, se manifeste dans le goût de Busoni pour le cérémonial: «Renouant avec les anciens mystères, l'opéra devrait prendre la forme d'une cérémonie semi-religieuse, qui rompe avec le quotidien, élève le spectateur tout en le stimulant et en le divertissant. $»^{44}$

Ce n'est pas un hasard si l'œuvre que Busoni plaçait au-dessus de toutes les autres et dans laquelle il voyait l'opéra par excellence était $L a$ Flûte enchantée. Certains passages de Turandot semblent vouloir rendre hommage au chef-d'œuvre de Mozart, notamment la scène d'entrée d'Altoum au premier acte (aria et dialogue, $\mathrm{n}^{\circ} 4$ et 5) : l'aria en mi majeur dans laquelle il s'en remet à Confucius pour qu'il lui donne un fils et qu'il lui permette de rejoindre ses ancêtres rappelle par sa solennité, ses longues phrases mélodiques et par sa tessiture extrêmement grave l'aria "In diesen heil'gen Hallen » (elle aussi en mi majeur) chantée par Sarastro au second acte de La Flûte enchantée. Mais c'est surtout dans la scène (dans laquelle alternent arioso et dialogues parlés) entre l'empereur et Kalaf, venu pour se soumettre aux épreuves imposées par Turandot, que le parallèle avec l'opéra de Mozart est le plus évident : à la question d'Altoum, « Qui es- 
tu?», Kalaf répond: «Sire, un prince de conte», puis, lorsque son interlocuteur insiste : "Sire, non, je suis un prince, cela doit suffire ${ }^{45}$ ». Le spectateur ne peut alors s'empêcher d'y voir une allusion ironique aux fameuses paroles de Sarastro lorsqu'il présente la candidature de Tamino aux grands-prêtres d'Isis et d'Osiris et rétorque à l'Orateur qui annonce : « il est prince », en disant : « Plus encore, c'est un homme ${ }^{46}$. » Le bref solo de flûte qui fait suite à la réponse de Kalaf clôt avec humour cette série de références (on hésite à parler de parodie) à l'opéra de Mozart.

Turandot est à l'évidence conçue comme une tentative de réalisation pratique des théories esthétiques de Busoni, en même temps qu'on y découvre en filigrane une véritable poétique de l'opéra permettant de compléter et d'affiner les positions exprimées dans des textes tels que Pour une nouvelle esthétique musicale ou l'Ébauche de préface à la partition du Doktor Faust. On peut, pour reprendre une remarque d'Adorno ${ }^{47}$, voir dans l'idée de l'opéra selon Busoni telle qu'elle se manifeste dans Turandot une sorte de trait d'union entre la critique de Wagner par Nietzsche et le néoclassicisme de Stravinsky. Mais, curieusement, cette émergence du néoclassicisme passe par une réappropriation de la pensée musicale romantique, aboutissant à une conception de la musique qu'on pourrait qualifier de classico-romantique: dans son projet de rénovation de l'esthétique de l'opéra, le compositeur s'appuie ainsi en partie sur une esthétique empruntée à la première partie du $\mathrm{XIX}^{\mathrm{e}}$ siècle, à l'époque où ne s'était pas encore cristallisée l'opposition radicale entre le formalisme de Hanslick et le « réalisme » de Wagner et des « Nouveaux Allemands ».

\section{NOTES}

1. Ferruccio Busoni, Lettre à sa femme, 21.01.1913 : «Que penses-tu d'un opéra sur Turandot - en langue italienne, d'après Gozzi ?» (Busoni, Briefe an seine Frau, Leipzig / Zürich, Rotapfel Verlag, 1935, S. 271 : «Wie denkst du über Turandot als Oper - und zwar in italienischer Sprache, nach Gozzi ?»)

2. Voir : Antony Beaumont, Busoni the Composer, London, Faber and Faber, 1985, p. 241.

3. E.T.A. Hoffmann, Der Dichter und der Komponist, in Die Serapions-Brüder, Frankfurt a.M., Insel, 1983, Bd. I, S. 113 : «Denke an den herrlichen Gozzi. In seinen dramatischen Märchen hat er das ganz erfüllt, was ich von dem Operndichter verlange, und es ist unbegreiflich, wie diese Fundgrube vortrefflicher Opernsujets bis jetzt nicht mehr benutzt worden ist. »

4. E.T.A. Hoffmann, Kreisleriana, Stuttgart, Reclam, 1983, S. 26: «Die Musik schließt dem Menschen ein unbekanntes Reich auf, eine Welt, die nichts gemein hat mit der äußern Sinnenwelt, die ihn umgibt [...]». Cf. Ferruccio Busoni, Pour une nouvelle esthétique musicale : " On peut voir et entendre un orage sans l'aide de la musique; ce que la musique doit rendre compréhensible, c'est en revanche ce qui se passe pendant ce temps dans l'âme humaine, l'invisible et l'inaudible. » (Entwurf einer neuen Ästhetik der Tonkunst, ergänzte und kommentierte Neuausgabe von Martina Weindel, Wilhelmshaven, Florian Noetzel Verlag, 2001, S. 23: «Das Gewitter ist sichtbar und hörbar ohne Hilfe der Musik; was aber in der Seele des Menschen vorgeht, das Unsichtbare und Unhörbare, das soll die Musik verständlich machen. ») 
5. Ferruccio Busoni, Entwurf einer neuen Ästhetik der Tonkunst, op. cit., S. 24 : «Immer wird das gesungene Wort auf der Bühne eine Konvention bleiben und ein Hindernis für alle wahrhaftige Wirkung. »

6. Voir : Jean-François Candoni, «Le rôle de Carlo Gozzi dans la constitution de la dramaturgie wagnérienne ", in Carlo Gozzi entre dramaturgie de l'auteur et dramaturgie de l'acteur : un carrefour artistique européen, a cura di Andrea Fabiano, Ravenna, Longo Editore, 2007, p. 329-342.

7. Ferruccio Busoni, Ébauche de préface à la partition du Doktor Faust comprenant quelques remarques sur la possibilité d'un opéra, in De l'unité de la musique. Des tiers de ton au Jeune Classicisme. Des scènes, des bâtiments et des domaines connexes. Notes éparses (Entwurf eines Vorwortes zur Partitur des Doktor Faust enthaltend einige Betrachtungen über die Möglichkeiten der Oper,in Von der Einheit der Musik. Von Dritteltönen und Junger Klassizität. Von Bühnen und Bauten und anschließenden Bezirken. Verstreute Aufzeichnungen, kritische und kommentierte Neuausgabe, hrsg. von Martina Weindel, Wilhelmshaven, Florian Noetzel Verlag, 2006), S.107: "Während es für das Drama fast grenzenlose Möglichkeiten des Stoffes gibt, zeigt sich bei der Oper, daß nur solche Sujets ihr angemessen sind, die ohne Musik nicht bestehen, noch zum vollen Ausdruck gelangen könnten, die nach Musik verlangen und erst durch diese vollständig werden. »

8. Friedrich Schlegel, Geschichte der alten und neuen Literatur [1812-1814] (Histoire de la littérature ancienne et moderne), in Friedrich Schlegel, Kritische Schriften und Fragmente [1812-1814], hrsg. von Ernst Behler und Hans Eichner, Paderborn, Schöningh, 1988, Bd.4, S. 191: "In Gozzis fantastischen Volksmärchen, seinen Zauber- und Spektakelstücken, sehen wir eine wahrhaft poetische Erfindungskraft; aber ohne die musikalische Ausbildung, ohne den Schmuck der Fantasie, wodurch die Poesie, die in ihnen liegt, erst ganz zur Erscheinung und zur Wirkung kommen würde. »

9. Friedrich Schlegel, Fragments sur la littérature et la poésie [1797] (Fragmente zur Literatur und Poesie [1797]), in Friedrich Schlegel, Kritische Schriften und Fragmente [1798-1801], hrsg. von Ernst Behler und Hans Eichner, Paderborn, Schöningh, 1988, Bd. 5, S. 188.

10. August Wilhelm Schlegel, Sur l'art et la littérature dramatiques (1809) : "L'arbitraire de la représentation allait, dans la partie sérieuse de l'intrigue comme dans les lazzi qui l'accompagnaient, bien au-delà de la vérité naturelle. " (Über dramatische Kunst und Literatur, in Sämmtliche Werke, Bd. V, hrsg. von Eduard Böcking, Hildesheim, Olms, 1971 [reprint de l'édition de Leipzig 1846], S. 365 : «Die Willkür der Darstellung gieng in dem ernstaften Theile wie im beigesetzten Scherz weit über die natürliche Wahrheit hinaus. »)

11. Ferruccio Busoni, Zur Turandotmusik (1911), in Von der Einheit der Musik, op. cit., p. 63 : «Die Empfindung, daß es sich fortwährend - selbst bei den ans Tragische grenzenden Szenen - um ein Spiel handelt, fällt bei Schiller durchaus fort. $\mathrm{Zu}$ dieser Wirkung tragen vorzüglich die den Italienern vertrauten Maskenfiguren bei, die eine Brücke vom venezianischen Publikum in den fingierten Orient auf der Bühne schlagen und so die Illusion eines wirklichen Vorgangs wieder vernichten."

12. Voir: Helmut Feldmann, Die Fiabe Carlo Gozzis. Die Entstehung einer Gattung und ihre Transposition in das System der deutschen Romantik, Köln / Wien, Böhlau, 1971, S. 59.

13. On citera, exemple parmi tant d'autres, la réaction de Calaf au récit que lui fait Barach du comportement de Turandot envers ses prétendants : «Voilà encore cette vieille fable que j'ai entendue chez les habitants de Carazani, elle m'a fait rire. » (Carlo Gozzi, Turandot. Fiaba chinese teatrale tragicomica, in Fiabe teatrali, introduzione e note di Alberto Beniscelli, Milano, Garzanti, 1994, p. 128 : «Ecco l'antica / fiaba, che udii tra Carazani, e risi.») Les termes de «Fabel » et de «Spiel » apparaissent également de façon récurrente dans le livret de Busoni, en particulier au premier acte.

14. Friedrich Schiller, Über die ästhetische Erziehung des Menschen, in einer Reihe von Briefen, Fünfzehnter Brief, in Sämtliche Werke V, München, Hanser, 1959, S. 617-618 : « der Mensch soll mit der Schönheit nur spielen, und er soll nur mit der Schönheit spielen. » 
15. Ferruccio Busoni, Pour une nouvelle esthétique musicale (Entwurf einer neuen Ästhetik der Tonkunst, op. cit., S. 24-25 : « Es ergibt sich demnach eine kommende Möglichkeit in der Idee des übernatürlichen Stoffes. Und noch eine: in der des absoluten "Spieles", des unterhaltenden Verkleidungstreibens, der Bühne als offenkundige und angesagte Verstellung, in der Idee des Scherzes und der Unwirklichkeit als Gegensätze zum Ernste und zur Wahrhaftigkeit des Lebens. »)

16. Ferruccio Busoni, Turandot, Klavierauszug von Philipp Jarnach, Wiesbaden, Breitkopf \& Härtel, 1987, I, 2, n : « mein Leben spiel' ich gegen ein Gedicht. »

17. Friedrich Nietzsche, Le Cas Wagner (Der Fall Wagner, Stuttgart, Reclam, 1973, S. 98) : «Wagner est une névrose » [en français dans le texte].

18. Voir à ce sujet : Dieter Borchmeyer, Mozart ou la découverte de l'amour : « Le fait que l'on tombe amoureux d'un simple portrait n'est compréhensible, tout comme l'“amour par ouï-dire" (Niklas Luhmann), que si l'amour n'est pas encore quelque chose d'incommensurable, d'imprévisible, que s'il n'est pas encore situé dans l'individualité unique de l'autre aimé, mais dans des valeurs établies telles que la beauté, la vertu, la richesse, qui font que l'on aime. " (Mozart oder die Entdeckung der Liebe, Insel, Frankfurt a.M./ Leipzig 2005, S. 31 : «Daß man sich aufgrund eines bloßen Bildes verliebt, ist ebenso wie das "Lieben vom Hörensagen" (Niklas Luhmann) nur verständlich, wenn Liebe noch nichts Inkommensurables, Unkalkulierbares ist, noch nicht in der unverwechselbaren Individualität des geliebten Anderen, sondern in feststehenden Werten wie Schönheit, Tugend, Reichtum gründet, um derentwillen man liebt. »)

19. Ferruccio Busoni, Turandot, op. cit., I, $1, n^{\circ} 2$ : «Wie könnte ein Bildnis tödlich sein ?»

20. Ferruccio Busoni, Entwurf eines Vorwortes zur Partitur des Doktor Faust, op. cit., S. 107 : « Daß die sensuelle, oder "sexuelle" Musik (welche in einer Art Hartnäckigkeit, im Klangrausch besteht und so auf die Nervenklaviatur spielt) hier nicht am Platze ist, geht aus dem Wesen dieser Kunst, das rein abstrakt ist, eigentlich von selbst hervor. »

21. Ferruccio Busoni, Turandot, op. cit., I, 2, n% : « Tod oder Turandot. »

22. Ferruccio Busoni, Entwurf eines Vorwortes zur Partitur des Doktor Faust, op. cit., S. 109: «Das Schlagwort ersetzt in der Oper die "Tirade" des Dramas. »

23. Ferruccio Busoni, «Junge Klassizität " (Brief an Paul Bekker, 1920), in Von der Einheit der Musik, op. cit., S. 95 : « Ein Drittes ist [...] die Abstreifung des Sinnlichen und die Entsagung gegenüber dem Subjektivismus, (der Weg zur Objektivität - das Zurücktreten des Autors gegenüber dem Werke [...]).»

24. Ferruccio Busoni, Entwurf einer neuen Ästhetik der Tonkunst, op. cit., S. 22 : « Musik ist aber ein Teil des schwingenden Weltalls. »

25. Eduard Hanslick, Vom Musikalisch-Schönen, ein Beitrag zur Revision der Ästhetik der Tonkunst, Teil I : Historisch-kritische Ausgabe, Mainz, Schott 1990, S. 75 : « Tönend bewegte Formen sind einzig und allein Inhalt und Gegenstand der Musik. »

26. Ferruccio Busoni, Entwurf einer neuen Ästhetik der Tonkunst, op. cit., S. 14 : " "Absolute Musik" ist ein Formenspiel ohne dichterisches Programm, wobei die Form die wichtigste Rolle abgibt. Aber gerade die Form steht der absoluten Musik entgegengesetzt, die doch den göttlichen Vorzug erhielt zu schweben und von den Bedingungen der Materie frei zu sein."

27. Voir par exemple ces remarques figurant dans le chapitre V des Fantaisies sur l'art (1799) : «Sons, approchez-vous et sauvez-moi de ce douloureux désir terrestre de mots, enveloppez-moi de vos innombrables rayons et conduisez-moi vers vos éclatantes nuées, soulevez-moi jusqu'à l'immémoriale étreinte de l'amour céleste infini. » (Wilhelm Heinrich Wackenroder / Ludwig Tieck, Phantasien über die Kunst, Stuttgart, Reclam, 1973, S. 86-87 : « Kommt ihr Töne, ziehet daher und errettet mich aus diesem schmerzlichen irdischen Streben, wickelt mich ein mit euren tausendfachen Strahlen in eure glänzende Wolken und hebt mich hinauf in die alte Umarmung des alliebenden Himmels !») 
28. Dans son compte rendu À propos du concert radiophonique du 7 novembre 1930, Theodor W. Adorno précise sa pensée de la manière suivante: «C'est justement cette conscience de l'impossibilité de réaliser une norme et une objectivité, qu'il cherche en quelque sorte à opposer à la volonté d'expression débridée de l'individu musicien, qui le situe dans ce romantisme qu'il combat pourtant. Toute sa production se situe à la lisière du romantisme » (Zum Rundfunkkonzert vom 7. November 1930, in Adorno, Gesammelte Schriften, Bd. 18 [= Musikalische Schriften V], Frankfurt a.M., Suhrkamp, 1984, S. 559 : «Eben dies Bewußtsein der Unrealisierbarkeit einer kollektiven Verbindlichkeit und Objektivität, die er doch gleichwohl dem fessellosen Ausdruckswillen der musikalischen Person entgegenstellt, ordnet ihn der Romantik selber zu, die er bekämpft. Er steht in all seiner Produktion auf der Grenzscheide der Romantik [...]. »)

29. Voir à ce sujet : Antony Beaumont, Busoni the Composer, op. cit., p. 80 sq.

30. Voir: August Wilhelm Ambros, Die Grenzen der Musik und Poesie. Eine Studie zur Ästhetik der Tonkunst, Leipzig, Verlag von Heinrich Matthes, 1855, S. 36 ; S. 44.

31. Ibid., S. 77 : « Für Begebenheiten hat aber Musik keine Sprache.»

32. Ibid., S. 49 : «Die "tönende Arabeske" ist eben auch nur ein artiger Einfall, der bei näherer Prüfung wie eine Seifenblase zerplatzt.» («L'“arabesque sonore” n'est qu'une belle idée qui éclate comme une bulle de savon dès qu'on veut l'examiner de près. »)

33. Ibid., S. 21 : «Das geistige Moment des Kunstwerkes, das Idealmoment ist der vom Himmel geholte, zündende Prometheusfunke, der dem wohlgeformten, aber leblosen Thonbilde erst Leben gibt. "

34. Ferruccio Busoni, Turandot, op. cit., I, 2, $\mathrm{n}^{\circ} 8$ : « Ein Kuß der Erde von des Himmels Gunst. »

35. Carl Dahlhaus, Eine Ästhetik des Widerstands? Friedenstag von Richard Strauss, in Gesammelte Schriften 8, Laaber Verlag, 2005, S. 608 : «Von Wundern zu handeln ist dem musikalischen Drama, sofern es ein Drama aus dem Geiste der Musik zu sein versucht, gleichsam eingeboren. Die Theorie der Oper war immer schon, im 17. und 18. Jahrhundert ebenso wie in der Romantik von E.T.A. Hoffmann über Wagner bis zu Busoni, eine Ästhetik des "Wunderbaren", des "meraviglioso" - also des Gegensatzes zum "Wahrscheinlichen", das seit Aristoteles in der Poetik des gesprochenen Schauspiels gefordert wurde. »

36. Ferruccio Busoni, Turandot, op. cit., I, $2, n^{\circ} 8:$ : Was kriecht am Boden, fliegt gen Himmel, / Was tappt im Dunkeln, zündet Lichter [...]. »

37. Ibid. « Ich war betäubt. »

38. Ferruccio Busoni, Entwurf einer neuen Ästhetik der Tonkunst, op. cit., p. 10 : «Der Geist eines Kunstwerkes, das Maß der Empfindung, das Menschliche, das in ihm ist - sie bleiben durch wechselnde Zeiten unverändert an Wert; die Form, die diese drei aufnahm, die Mittel, die sie ausdrückten, und der Geschmack, den die Epoche ihres Entstehens über sie ausgoß, sie sind vergänglich und rasch alternd. »

39. Ferruccio Busoni, Turandot, op. cit., I, $2, \mathrm{n}^{\circ} 8$ : « darüber einsam Geweihte walten. »

40. Ferruccio Busoni, Entwurf eines Vorwortes zur Partitur des Doktor Faust, op. cit., S. 107 : «Es handelt sich bei der Oper um das musikalische "Gesamtkunstwerk"; im Gegensatz zum Bayreuther Gesamtkunstwerk. »

41. Ferruccio Busoni, Turandot, op. cit., I, 1, $n^{\circ} 3$ : « Barak, alles in ihr ist Einklang. »

42. Ibid., I, 2, $n^{\circ} 1$ : « Das ist des Kastraten Segen, / daß sein Geist bleibt überlegen. »

43. Ibid., II, 3, n5 : « "Tod, Tod oder Turandot", / und Weitres war aus ihm nicht zu locken!»

44. Ferruccio Busoni, Entwurf eines Vorwortes zur Partitur des Doktor Faust, op. cit., S. 107 : «An das alte Mysterium wieder anknüpfend, sollte die Oper zu einer un-alltäglichen, halb-religiösen, erhebenden, dabei anregenden und unterhaltsamen Zeremonie sich gestalten. »

45. Ferruccio Busoni, Turandot, I, 2, $\mathrm{n}^{\circ} 5$ : «ALTOUM: Wer bist du? / KALAF: Sire, ein Menschenprinz [...] Sire, nein, ich bin ein Prinz : es genüge.»

46. W.A. Mozart / E. Schikaneder, Die Zauberflöte, II, 1 : « SPRECHER : Er ist Prinz ! - / SARASTRO : Noch mehr - er ist Mensch!» 
47. Theodor W. Adorno, Dix-neuf contributions sur la nouvelle musique: "Le néoclassicisme a été préparé littérairement par le combat de Nietzsche contre Wagner et par les textes de Busoni et de Cocteau ("Le Coq et l'Arlequin", 1919), qui renvoient tous deux à Nietzsche.» (Neunzehn Beiträge über neue Musik, in Theodor W. Adorno, Gesammelte Schriften, Bd.18 [= Musikalische Schriften V], Frankfurt a.M., Suhrkamp, 1984, S. 86: «Der Neoklassizismus war literarisch vorbereitet durch den Kampf Nietzsches gegen Wagner und durch die Schriften von Busoni und Cocteau ("Le Coq et l'Arlequin", 1919), die beide auf Nietzsche zurückgehen. »)

\section{RÉSUMÉS}

Turandot, opéra de Ferruccio Busoni adapté d'une fiaba teatrale de Carlo Gozzi, est ici mis en perspective avec les différents écrits esthétiques du compositeur, ce qui permet en même temps de le lire comme une réflexion métaphorique sur la fonction de la musique et de l'art en général. L'article tente de montrer comment la poétique de l'opéra qui sous-tend Turandot procède d'une volonté de renouveler le genre en se détournant à la fois du vérisme italien et du modèle wagnérien, qui ne sont pour Busoni que deux faces d'une même réalité. Paradoxalement, le compositeur met ici en place ce qu'il appelle le «jeune classicisme» en renouant avec le romantisme d'E.T.A. Hoffmann, lu à travers le prisme d'un néo-classicisme inspiré des théories d'August Wilhelm Ambros.

Ferruccio Busonis Oper Turandot, nach der gleichnamigen fiaba teatrale Carlo Gozzis, wird hier im Lichte der musiktheoretischen Schriften des deutsch-italienischen Komponisten analysiert, wobei das Libretto als metaphorische Reflexion über Musik und Kunst überhaupt verstanden wird. Die Erneuerung der Gattung, die dem Komponisten der Turandot vorschwebte, geht mit einer radikalen Abkehr vom italienischen Verismus und vom Wagnerschen System einher, welche als verwandte Kunstansätze entlarvt werden. Merkwürdig genug entsteht Busonis „Junge Klassizität" auf dem Umweg über eine weitgehend romantische, auf E.T.A. Hoffmann zurückgehende Musikphilosophie, wenngleich das romantische Moment durch das Prisma der Theorien August Wilhelm Ambros' rekonstruiert wird.

\section{INDEX}

Mots-clés : poétique de l'opéra, Fiaba teatrale

\section{AUTEURS}

\section{JEAN-FRANÇOIS CANDONI}

Université de Paris IV-Sorbonne 